\title{
Todesanzeige.
}

Am 7. Nov. I9I3 verschied zu Chyrow in Galizien P. Alexander Gromadzki S. J. Zu Sielec unweit Kowel (Gouv. Volhynien, Rußland) am 20. März 1833 geboren, absolvierte er das Gymnasium zu Rowno im Jahre 1855 und die Universität Kiew im Jahre 1860 (Febr.). Während der Jahre $1860-1878$ bekleidete er die Stelle eines jüngeren, dann eines älteren Astronomen-Observators an den Sternwarten Kiew, Pulkowa und Moskau (1869-1878). Gegen das Ende seines Aufenthaltes in Pulkowa, nämlich 1869, erlangte er an der Universität Petersburg den Grad eines Magisters der Astronomie und Mechanik.

Aus unbekannten Gründen verließ Gromadzki im Jahre 1878 den russischen Staatsdienst, um in den Jesuitenorden einzutreten und machte damit seiner wissenschaftlichen Laufbahn ein Ende; er wirkte später (seit 1883 ) als ein wegen seiner Güte von den Schülèrn vielgeliebter Lehrer der Mathematik und Physik am Jesuitengymnasium zu Tarnopol, dann zu Chyrow in Galizien.

Die wissenschaftliche Tätigkeit Gromadzkis war dem Gebiete der beobachtenden, praktischen Astronomie zugewandt. Dahin gehört seine Magister-Dissertation: „Über die Fehler der Meridiankreise* (St. Petersburg 1869, russ.), sowie seine anderen Arbeiten. Der I. Band der "Annales de l'Observatoire de Moscou " wurde zum größten Teil von ihm bearbeitet, die Bände 2 und 3 enthalten seine Beobachtungen des Kometen von Coggia und des Planeten Mars, seine Untersuchungen der Fehler der Kreisteilungen des Moskauer Meridiankreises, ferner der Thermorneterfehler. Im 4. Band befinden sich zwei Abhandlungen, die zwar auf Gromadzkis Beobachtungen beruhen, aber von anderen geschrieben wurden. Der 6. Band enthält seine Untersuchungen betreffend Bestimmung des thermometrischen Koeffizienten der Refraktion. Die Bände 69, 84 und 85 der A. N. enthalten einige Notizen über die Beobachtungen Gromadzkis. M. P. Rudzki.

\section{Elliptische Elemente des Kometen 1911 V (Brooks).}

Bei der Inangriffnahme der definitiven Bahnbestimmung des Kometen I 9 I I V (Brooks) stellte sich als erstes Erfordernis die Kenntnis guter genäherter Elemente heraus. 'Das bisher beste, d. h. den größten heliozentrischen Bogen begreifende Elementensystem ist das parabolische von Millosevich A. N. 4536. Die nach demselben gerechnete Ephemeride weicht aber an manchen Stellen um mehrere Bogenminuten von der wirklichen Bahn ab. Ich beschloß daher, mir ein besseres System zư verschaffen, und wählte aus den bereits veröffentlichten Beobachtungen $3^{1}$ aus, die ich zu 8 Normalörtern vereinigte. Die benutzten Beobachtungen reichen von I 9 I I Juli 2 I bis I 9 I 2 Febr. 27, umfassen also einen Zeitraum von ca. 220 Tagen und einen heliozentrischen Bogen von $245^{\circ}$. Ich bemerke vorweg, daß bei der späteren Ausgleichung alle Normalorte gleiches Gewicht erhielten, obwohl sie aus einer sehr verschiedenen Anzahl von Beobachtungen erhalten waren. Die Herstellung der wahrscheinlichsten Parabel durch Variation des Verhältnisses der geozentrischen Distanzen zeigte nir die Unzulänglichkeit der Parabel, da diese Restfehler von mehreren Bogenminuten bestehen ließ. Ich suchte infolgedessen den wahrscheinlichsten Kegelschnitt zu ermitteln und fand durch Variation der geozentrischen Distanzen folgende meinen Zwecken genügende elliptischen Elemente ${ }^{1}$ ):

$$
\begin{aligned}
& T=\text { I9 I I Okt. } 27.77555 \mathrm{~m} . \mathrm{Z} \text {. Berlin } \pm 0 \text { door } 42 \\
& \omega=153^{\circ} \mathrm{I}^{\prime} 27^{\prime 2} 2 \\
& \delta=2925657.1\} \text { m. Ekl. 1911.0 } \pm 4.4 \\
& i=334812.8\} \quad \pm 0.1 \\
& \log q=9.689688 \\
& e=0.99704
\end{aligned}
$$

Um zu zeigen, daß der elliptische Charakter der Bahn reell ist, führe ich neben den $\mathrm{m}$. F. noch die Darstellung der 8 zugrunde gelegten Orte durch meine elliptischen Elemente an:

$$
\begin{array}{crc|ccr}
\text { Normalort } & \Delta \lambda \cos \beta & \Delta \beta & \text { Normalort } & \Delta \lambda \cos \beta & \Delta \beta \\
\text { I } & 0.0 & 0.0 & \text { V } & +1.4 & +2.7 \\
\text { II } & -8.6 & -7.5 & \text { VI } & +2.8 & -3.6 \\
\text { III. } & -3.7 & +8.4 & \text { VII } & 0.0 & 0.0 \\
\text { IV } & -8.1 & -1.4 & \text { VIII } & +1.4 & +2.5
\end{array}
$$

Wenn man in Betracht zieht, daß diese Elemente ohne Berücksichtigung der Störungen sowie ohne besondere Kritik der Beobachtungen und Vergleichsternpositionen erhalten sind, so kann man sie zufolge ihrer Übereinstimmung mit den Beobachtungen als vorläufig genügend erachten. Die größeren Abweichungen in $\lambda$ bei II und IV sowie in $\beta$ bei II und III prägten sich schon in den Beobachtungen durch mangelnde innere Übereinstimmung aus.

Wie oben bereits angedeutet, beabsichtige ich, durch Verwendung aller vorhandenen Beobachtungen das obige sehr genäherte Elementensystem des Kometen I 9 I I V zum definitiven $z u$ verbessern. Ich richte daher an alle Herren Beobachter die ergebene Bitte, etwa noch unveröffentlichte Beobachtungen dieses Kometen recht bald bekannt zu geben, oder mir direkt an meine untenstehende Adresse mitzuteilen.

Königsberg, Pr., Goltzallee 28 B, I 9 I 3 Nov. 11.

\section{E. E. Kühne.}

1) Die oskulierende Umlaufszeit beträgt ca. 2000 Jahre.

Kometenbahnen. Definitive Bahnbestimmungen werden ausgefuihrt: Von Prof. C. Wirtz, Straßburg, für den Kometen I766 II; von Frau Terentjewa und Frl. Martjanowa, St. Petersburg, für den Kometen I 9 Io IV (Metcalf); von Herrn L. Lindsey, Syracuse, für den Kometen I9 I II (Kiess) und von Herm F. Balanowsky, Pulkowo, für den Kometen r9r3 C (Neujmin). Die Berechner bitten um baldige Mitteilung etwa noch nicht veröffentlichter Beobachtungen der neueren Kometen.

\footnotetext{
Inhalt zu Nr. 4701. P. Guthnick. Nachweis der Veränderlichkeit des kurzperiodischen spektroskopischen Doppelsterns $\beta$ Cephei mittels photoelektrischet Messungen, 357. - E. Grossmann. Ein selbstschreibender Ableseapparat. 365. - F. Kramer. Zur Frage empirischer Elementenkorrektionen. 367. - Mitteilungen uber Kleine Planeten. 369. $-M$. $P$. Rudzki. Anzeige des Todes von Alexander Gromadzki. 371. - E. E. Kühne. Elliptische Elemente des Kometen I91 I V (Brooks). 371. - Kometenbahnen. 371. 